\title{
Improving Trainees' Experience During The Implementation Of Out Of Hours' Rotas
}

\author{
Gareth Garrett, Maheera Cheema \\ Camden and Islington NHS Foundation Trust
}

\begin{abstract}
During clinical induction for psychiatry at Camden and Islington NHS Foundation Trust, doctors covering out of hours rotas are expected to attend a meeting to finalise on call duties. The meeting aims to support doctors to utilise leave whilst ensuring patient safety. The meeting has being cited as being a negative experience for doctors. Attendees were asked to complete a questionnaire before and after the introduction of a new policy that would prepare doctors adequately and ensure the meeting runs smoothly. Post intervention, there was an improvement in the experience and satisfaction of doctors who attended.
\end{abstract}

\section{Problem}

Camden and Islington NHS Foundation Trust delivers mental health services to people living in the London Boroughs of Camden and Islington. It provides treatment to around 30,000 patients per year. The trust commissions liaison psychiatry services in three large hospitals within these boroughs. This project focused on trainees providing out of hours' care at University College Hospital.

Psychiatry trainees are an essential component of liaison psychiatry services out of hours.

The trust endorses a rota meeting as part of doctors' induction into new clinical posts. The meeting functions to ensure all out of hours' shifts are filled at the start of the rotation, whilst providing trainees with an open forum to swap shifts. It also ensures that there is adequate medical cover to all inpatient wards during a normal working week.

The meeting takes place biannually on the first day of each new sixmonth rotation. It is scheduled to last three hours and is attended by doctors in psychiatry who are in their first three years of speciality training. It is chaired by a Consultant Psychiatrist, and there is an expectation that all annual leave and study leave is published on the rota produced at the end of the meeting.

Such a process whilst admirable in it's objectives could be viewed as being overly paternalistic by attendees, and could act as a deterrent for adequate notice for on call duties.

The aim of this project was to positively affect the meeting to better the experience of attendees.

\section{Background}

It is known that poor arrangements for rotas, cover, and leave can have a negative impact on doctors' morale and health.[1] Giving more control to doctors over when they undertake out of hours' work could reduce fatigue, and therefore enhance patient care.[2] It has been shown that low job control is associated with high levels of work and family life interference.[3] It is likely therefore that any intervention to promote greater flexibility of working hours would have a positive impact on an employee's morale. In regards to the potential link between first impressions of a clinical post and the subsequent effect on doctors' morale, a thorough literature search did not reveal any relevant findings.

The British Medical Association recommend six weeks' notice in the publishing of on call shifts on out of hours' rotas. It is common practice that shifts are swapped between colleagues in order that doctors can utilise annual and study leave. The rota meeting acts as a forum such that all swaps can be made and a safe out of hours' rota published.

We were informed by Consultant colleagues that the meeting was introduced after a previous cohort of psychiatric trainees were affected by an unprecedented amount of sickness. It was subsequently unsafe for the remaining doctors to take time off from clinical duties.

The success of the rota meeting can be measured not only by the final rota produced, but also by the experience of attendees, who may be new to the trust or to psychiatry as a speciality.

\section{Baseline measurement}

All doctors covering the University College Hospital out of hours' rota from February 2015 to August 2015 were asked to complete a questionnaire about their experience of the meeting. The questionnaire generated a mixture of qualitative and quantitative data regarding the experience of attendees before, during, and after the meeting. There were also questions to identify whether this was a doctor's first experience of the meeting and whether they were new to psychiatry.

The baseline measurement was obtained via an online survey using a well-known free access platform. The survey ran for the week following the meeting, 4th to 11 th February 2015 inclusive. The 
survey was distributed via attendees' work email.

Ten attendees responded to the initial survey which represented $71 \%$ of the cohort. Of the respondents, $70 \%$ had had previous experience of the meeting and $30 \%$ were new to psychiatry. Most doctors $(70 \%)$ were able to attend the whole meeting. The majority of respondents $(70 \%)$ found the meeting unstructured but generally supportive. It was widely agreed $(90 \%)$ that the meeting benefited from a consultant presence. Only $40 \%$ of attendees felt the overall process was satisfactory, with $20 \%$ citing the final rota as unsatisfactory with regards to training needs and patient safety.

Qualitative feedback showed that most trainees were unaware of the recommendation to have all requests for leave ready at the meeting. The atmosphere was described as tense, anxious, and pressured. After the meeting attendees reported feeling anxious, drained, and fatigued, and there was a feeling the process was unfair.

\section{Design}

After considering areas for improvement it was decided a policy for the implementation for future meetings be written. It was agreed that the responsibility should be with the current trainee representatives to prepare future cohorts of doctors adequately so that future meetings run efficiently and were a positive experience for attendees.

Doctors would need adequate notice to attend, would require contact details for all attendees and would be politely requested to consider all leave requirements prior to the meeting. In addition, attendees would be given an agenda, an explanation for the meeting's existence and the positive contributions of the meeting highlighted e.g. a mechanism to honour leave requirements and generate a rota ensuring patient safety.

\section{Strategy}

PDSA Cycle 1.

We predicted trainees' experience of the meeting could be improved upon. Results from the baseline questionnaire were presented at the local junior senior's meeting. Each aspect of trainees' experience was discussed and areas for improvement considered. A draft policy for future practice was circulated to current trainee representatives. Personal assistants to the medical directorate and colleagues in human resources were consulted to ensure any policy generated was not judged to be unreasonable for non-medical colleagues. The policy was implemented for the next cohort of doctors covering the new rota from August 2015 to February 2016. A repeat questionnaire was undertaken following the subsequent rota meeting from 5th to the 12th August 2015 inclusive. Overall the findings were positive but allowed for areas of improvement.

PDSA Cycle 2.
The results of the first PDSA cycle were circulated to trainees. The importance of the policy was reiterated to the new trainee representatives, and defined within their job description. One of the aims of this was to ensure quality was maintained after the authors moved onto different clinical posts. The policy was implemented successfully for the cohort covering the rota from February 2016 to August 2016. A repeat questionnaire was circulated from 3rd to 10th February 2016 inclusive following the respective rota meeting. Findings were positive once more.

\section{Results}

Ten doctors responded to the questionnaire in PDSA 1 which represented $83 \%$ of the cohort. Of the respondents, $60 \%$ had had previous experience of the meeting and $20 \%$ were new to psychiatry. Most doctors (90\%) were able to attend the whole meeting. All respondents found the meeting to be structured and $90 \%$ found colleagues supportive. It was again agreed $(90 \%)$ that the meeting benefited from a consultant presence. Most $(80 \%)$ attendees felt the overall process was satisfactory, with $100 \%$ citing the final rota as satisfactory with regards to training needs and patient safety.

Qualitative feedback from the first cycle showed that respondents felt prepared for the meeting. The atmosphere was described as optimistic and pleasant. After the meeting attendees reported feeling fatigued but satisfied.

The results from the questionnaire in the second PDSA cycle were also positive. Eight doctors responded representing $80 \%$ of the cohort. Of the respondents $75 \%$ had had previous experience of the meeting and $25 \%$ were new to psychiatry. All respondents were able to attend the whole meeting and it was commented upon that the meeting ran smoothly and was significantly shorter than usual. A consultant presence was felt to be beneficial by $75 \%$ of attendees. Respondents found the meeting both structured (100\%) and supportive (100\%). Respondents found the process satisfactory $(100 \%)$ as well as the final rota $(100 \%)$.

Qualitative feedback revealed the meeting as being a better experience when given adequate preparation. It was commented upon that the meeting was a good opportunity to meet with and be supportive to colleagues.

\section{Lessons and limitations}

This project highlighted the importance of first impressions to a clinical post, and how this may impact on a doctor's experience. The project showed that a meeting to support the safe implementation of out of hours' cover can be worthwhile, but that adequate communication is essential to make it a positive experience for attendees.

In regards to limitations it should be noted that there is a large burden of responsibility placed on the outgoing trainee representatives to adequately prepare the incoming cohort of doctors. The rota meeting is unique to Camden and Islington NHS 


\section{BMJ Quality Improvement Reports}

Foundation Trust and may therefore not be applicable in other clinical settings. The sample size although small represents the whole cohort of doctors affected. It is not clear whether a particularly positive or negative experience of the meeting would have affected a doctor's readiness to respond to the questionnaires. Our strategy does not address other factors that could affect attendees' experience of the meeting, including group dynamics and personal factors.

We would expect that the improvements we have observed will be maintained over time. The project will be highlighted to new trainee representatives as part of their job description as described in PDSA cycle 2. We do not intend to make the project a mandatory part of their role, but would welcome repeat cycles should representatives feel the process could be further improved, or if quality control is needed.

\section{Conclusion}

The positive attributes of the rota meeting are numerous and were reflected in our results both pre and post intervention. One of the functions of the meeting is to produce a safe and fully staffed rota. This was achieved in each of the meetings we observed. We did not analyse data on the number of absences that affected each cohort over the following six months. This could be a focus for future projects.

The baseline measurement data highlighted that many trainees had a negative experience which may have impacted their morale and enthusiasm entering into the new clinical post. Work conditions, hours of work, and changes to the NHS were the three biggest factors affecting morale according to a BMA survey in 2014.[4] Through a few simple interventions, post measurement data showed trainee's experience to be greatly improved.

\section{References}

1 Lambert T, Smith F, Goldacre M. Doctors' views about their work, education and training three years after graduation in the UK: questionnaire survey. JRSM open, Dec 2015, vol. 6, no. 12, p. 2054270415616309., 2054-2704

2 Tucker P, Bejerot E, Kecklund G, Aronsson G, Åkerstedt T. The impact of work time control on physicians' sleep and well-being Applied ergonomics, Mar 2015, vol. 47, p. 109-116, 1872-9126

3 Heponiemi T, Elovainio M, Pekkarinen L, Sinervo T, Kouvonen A. The effects of job demands and low job control on work-family conflict: the role of fairness in decision making and management. Journal of Community Psychology, Apr 2008 Vol. 36, Issue 3, p. 387-398

4 http://bma.org.uk/news-views analysis/news/2014/september/doctors-report-low-morale. Accessed on 13th March 2016.

\section{Declaration of interests}

Nothing to declare

\section{Acknowledgements}

None

\section{Ethical approval}

Local policy meant that ethical approval was not required. 